\title{
Case Report \\ Intrathoracic Giant Mass with Anomalous Vascular Origin in an Asymptomatic Patient: A Case Report
}

\author{
Michela Solinas *, Valentina Conti and Fabrizio Grignani \\ General Thoracic Surgery, Civil Hospital of Legnano, 20025 Milan, Italy; valentina.conti@asst-ovestmi.it (V.C.); \\ fabrizio.grignani@asst-ovestmi.it (F.G.) \\ * Correspondence: michela.solinas@asst-ovestmi.it
}

\begin{abstract}
Diagnostic and surgical difficulties were presented in case of a giant mass, found incidentally, in a 65-year-old healthy and asymptomatic man. The patient underwent different diagnostic procedures. Surgery was the treatment of choice. A combination of both open technique and videothoracoscopy to reduce the risk of seeding, pending a certain diagnosis, was resolutive. The postoperative period and recovery time were uneventful. The patient was discharged on the 5 th postoperative day. The histological diagnosis was benign schwannoma. There are different aspects to think about, based also on the experience of each center. According to our experience, this is a rare situation; the combined surgical techniques to control the vascular risk and tissue infiltration of the mass seemed to us the approach of choice.
\end{abstract}

Keywords: giant schwannoma; videothoracoscopy; surgery; thoracotomy; benign tumor; giant mass; abnormal vascularization

check for updates

Citation: Solinas, M.; Conti, V.; Grignani, F. Intrathoracic Giant Mass with Anomalous Vascular Origin in an Asymptomatic Patient: A Case Report. Surgeries 2022, 3, 1-3. https://doi.org/10.3390/ surgeries3010001

Academic Editor: Cornelis F.M. Sier

Received: 28 September 2021

Accepted: 10 December 2021

Published: 22 December 2021

Publisher's Note: MDPI stays neutral with regard to jurisdictional claims in published maps and institutional affiliations.

Copyright: (C) 2021 by the authors. Licensee MDPI, Basel, Switzerland. This article is an open access article distributed under the terms and conditions of the Creative Commons Attribution (CC BY) license (https:// creativecommons.org/licenses/by/ $4.0 /)$.

\section{Introduction}

Schwannoma is the most common mediastinal neurogenic tumor (50\%). Generally, the main localizations are the head and neck. Thoracic schwannomas make up $9 \%$ of neurogenic tumors and they are usually localized in the posterior mediastinum, because of their origin from roots of intercostal nerves [1]. The peak incidence is between the third and fifth decades of life. They affect men and women equally and, most of the time, they are asymptomatic [2]. When symptoms appear, they are related to invasion of intercostal nerves, bones, chest wall, or the tracheobronchial tree [2]. A chest x-ray for an unrelated cause is often the way they are diagnosed. Computed tomography (CT) is useful to determine the exact location in the mediastinum and invasion of adjacent structures [1]. Higher-grade radiological images are obtained with magnetic resonance imaging (MRI), especially with regard to vascular structures $[1,3]$. Total resection is considered the treatment of choice and it can be achieved through several combined thoracic-neurosurgical approaches, that are not without consequences [4]. Fortunately, in recent years, minimally invasive techniques have been developed and they have been increasingly used [4]. However, it is not always possible. Up to now, there are 10 cases dealing with a giant mediastinal schwannoma in the literature [5]. This case report shows the usefulness of the combination of the traditional surgical approach and videothoracoscopy to remove a giant schwannoma in a 65-year-old heathy and asymptomatic patient, without serious consequences, as can happen with the traditional technique alone.

\section{Case Presentation}

The patient was seen after a routine chest x-ray. The imaging showed a giant opacity of the left lung. No cardiological or respiratory problems were reported by the patient. He had always been healthy. A chest $\mathrm{CT}$ scan confirmed the presence of a mass of $11 \mathrm{~cm} \times 17 \mathrm{~cm} \times 13 \mathrm{~cm}$ with adhesion to the III-IV-V intercostal spaces. It was well capsulated and pressed against almost the entire lung with dislocation, but not infiltration, 
of mediastinal structures. Its perfusion derived from a vessel of the anterior wall of the descending aorta (Figure A1). The radiological report was unclear and did not differentiate the diagnosis between giant lymphoma, mesothelioma, less invasive sarcoma, or schwannoma. Because of the radiological uncertainty, fine-needle biopsy was performed, but it was not diagnostic. Based on the data collected so far, isolation of the mass was difficult, because of the involvement of almost the whole cavity and because of its size, which made it difficult to move. Therefore, the established procedure was to perform a left thoracotomy in the $\mathrm{V}$ intercostal space and to continue by extra-pleural approach to obtain a radical excision. Furthermore, thoracoscopic access in the VIII intercostal space and the introduction of $10 \mathrm{~mm}$ optics were useful for the identification of the vascular peduncle, which was controlled with clips. Through the thoracoscopic access, the real tissue involvement of the mass was also clear. The giant mass was partially attached to the diaphragm and the lung. The diaphragmatic surface layer was partially removed, without the need to reinforce with prostheses or suturing, while a mechanical stapler was used to isolate the healthy lung tissue. The visceral pleura was macroscopically healthy. Fortunately, no other major structures were involved. Cytologic exams, taken in different areas of the mass, revealed a possible polycystic structure. In fact, some areas were full of clear citrine yellow liquid, while other ones revealed a solid consistency. However, cytology was also not useful to obtain an intraoperative diagnosis, although it did not change the therapeutic path undertaken. Once the mass had been removed, two 28Ch chest tubes were placed and connected to a Medela ${ }^{\mathrm{TM}}$ system at $-20 \mathrm{~cm} \mathrm{H}_{2} \mathrm{O}$. The patient was transferred to the Intensive Care Unit (ICU), as a precautionary measure.

\section{Results}

The patient was monitored in ICU for almost $24 \mathrm{~h}$; the left lung was completely expanded at the postoperative chest $\mathrm{x}$-ray. The recovery was uneventful and pain control was optimal, so the patient was discharged on the 5th post-operative day. The histology was benign schwannoma.

\section{Discussion and Conclusions}

Different aspects appear. The very indolent growth of a giant schwannoma allowed the patient to adapt and be completely asymptomatic, although mediastinal deviation was present. The vascularization origin was atypical; the vascular peduncle derived from the anterior wall of the descending aorta; it was an ectopic vessel with its own origin. It was not, therefore, an intercostal vessel or a derivative. The mass radiologically mimicked a malignant tumor, with the consequent decision for direct surgical excision. MRI was not considered necessary, because vascular structures were well evident on CT scan and it would not have led to variations in the therapeutic path. Despite initial suspicion of a malignant tumor, neoadjuvant therapy was not considered, because of the lack of a pre-operatively certain diagnosis. In fact, although radiological images suggested a malignant disease, the cyto-histologic pattern was not diagnostic. Therefore, direct surgical approach was a joint decision, even at the cost of a possible demolitive surgery. In this case, a combined surgical approach, with videothoracoscopy and open thoracotomy, was a winning choice. This association allowed one to proceed by the extrapleural way in safety, always under vision, without resecting the ribs. Therefore, the procedure was sure, with reduced risk of recurrence, less extensive and painful, and it was a one-stage excision.

Author Contributions: Conceptualization, M.S and F.G.; methodology, M.S.; software, M.S.; validation, M.S., V.C. and F.G.; formal analysis, M.S.; investigation, M.S.; resources, M.S.; data curation, M.S.; writing — original draft preparation, M.S.; writing — review and editing, M.S.; visualization, M.S.; supervision, F.G.; project administration, M.S.; funding acquisition, no one. All authors have read and agreed to the published version of the manuscript.

Funding: This case report received no external funding.

Institutional Review Board Statement: Not applicable. 
Informed Consent Statement: Not applicable. Verbal informed consent was obtained by the patient.

Data Availability Statement: Not applicable. Verbal informed consent was obtained by the patient.

Conflicts of Interest: The authors declare no conflict of interest.

\section{Appendix A}

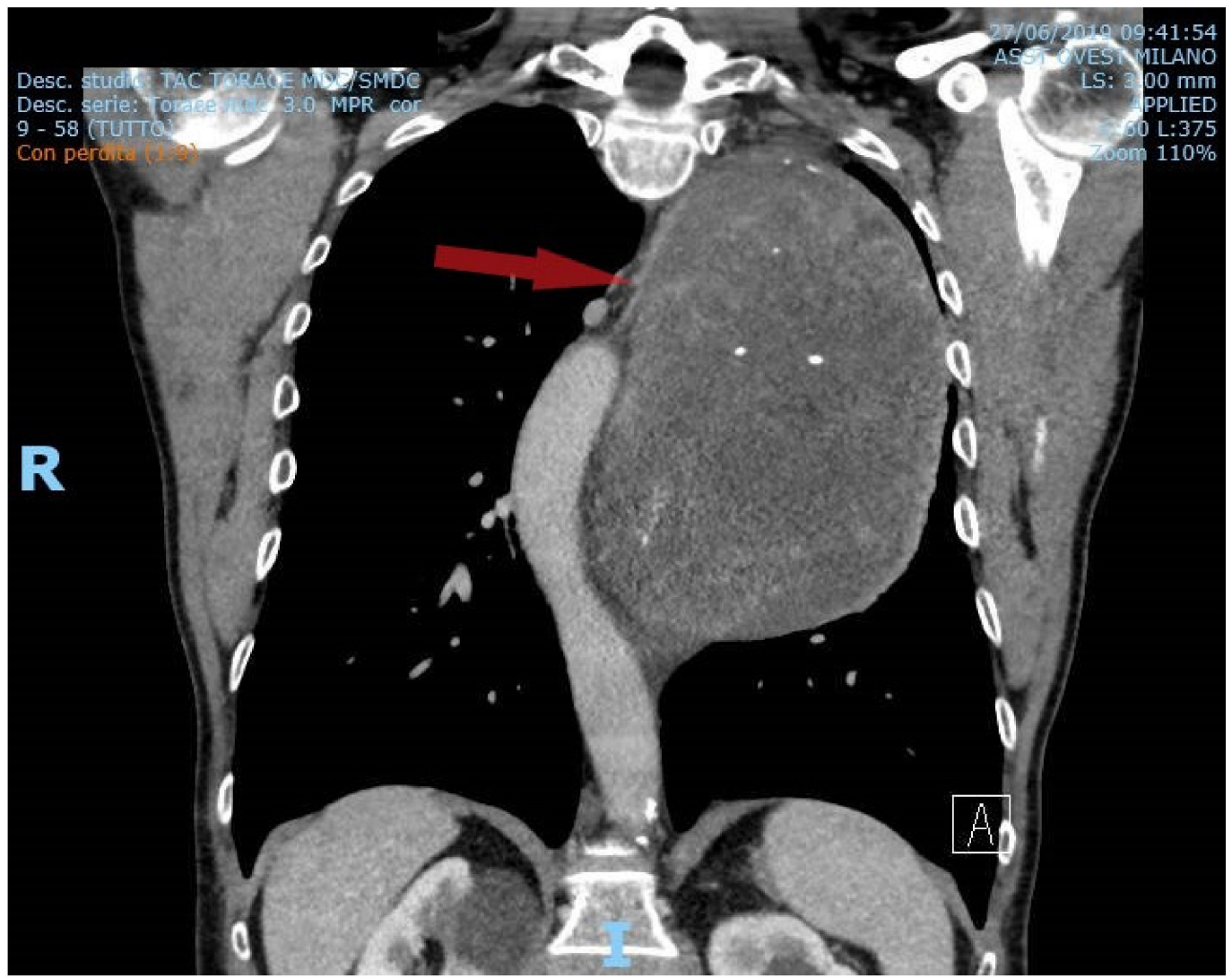

Figure A1. The arrow indicates the ectopic vessel nourishing the mass and the mediastinal deviation with compression of the descending aorta.

\section{References}

1. Kapoor, A.; Singhal, M.K.; Narayan, S.; Beniwal, S.; Kumar, H.S. Mediastinal schwannoma: A clinical, pathologic, and imaging review. South Asian J. Cancer 2015, 4, 104-105. [CrossRef] [PubMed]

2. Amin, R.; Waibel, B.H. An unusual presentation of a posterior mediastinal schwannoma associated with traumatic hemothorax. Case Rep. Surg. 2015, 2015, 175645. [CrossRef] [PubMed]

3. Laurent, F.; Latrabe, V.; Lecesne, R.; Zennaro, H.; Airaud, J.Y.; Rauturier, J.F.; Drouillard, J. Mediastinal masses: Diagnostic approach. Eur. Radiol. 1998, 8, 1148-1159. [CrossRef] [PubMed]

4. Corrivetti, F.; Stati, G.; Carpineta, E.; Sufianov, A.; Mastronardi, L. Minimally Invasive Microsurgical Removal of Giant Dumbbell Thoracic Neurofibroma: Usefulness of Current Technology for Minimizing the Approach-Illustrative Case and Technical Video. World Neurosurg. 2021, 147, 157. [CrossRef]

5. Mubashir, M.; Salam, A.; Sonawalla, A.; Fatima, H.; Fatimi, S.H. Rare Presentation of a Posterior Mediastinal Cystic Schwannoma as a Large Pleural Effusion. Cureus 2017, 10, e1558. [CrossRef] 